\title{
Robust control of PGD-based numerical simulations
}

\author{
Ludovic Chamoin* and Pierre Ladevèze \\ LMT-Cachan (ENS Cachan - CNRS - Paris 6 University), 61 Avenue du Président Wilson, Cachan \\ Cedex 94235, France
}

\begin{abstract}
In this paper, we develop an error estimator that enables to control effectively the quality of numerical solutions obtained using proper generalised decomposition. The method is based on the Constitutive Relation Error and the construction of associated admissible fields. It takes all error sources (discretisations, truncation of the modal representation, etc.) into account and can be used, introducing adjoint-based techniques, for goal-oriented error estimation. Furthermore, specific indicators can be derived to split error contributions and thus drive adaptive procedures in an optimal manner.
\end{abstract}

Dans ce travail, nous développons une méthode d'estimation d'erreur qui permet de contrôler efficacement la qualité des solutions numériques obtenues par la PGD. L'estimateur associé est basé sur la notion d'Erreur en Relation de Comportement et s'appuie donc sur une solution admissible. Il prend en compte toutes les sources d'erreur (discrétisations en espace et en temps, troncature de la représentation modale, ...) et peut être utilisé, conjointement aux techniques de l'adjoint, pour le contrôle d'erreur sur des quantités d'intérêt. De plus, des indicateurs spécifiques permettent de séparer les contributions des différentes sources afin de pouvoir conduire de façon optimale des procédures adaptatives.

Keywords: verification; model reduction; PGD; error estimation; constitutive relation error

Mots-clés: vérification; réduction de modèle; PGD; estimation d'erreur; erreur en relation de comportement

\section{Introduction}

Among all model reduction methods which are available nowadays, the proper generalised decomposition (PGD) is very promising and is the object of numerous research works (Chinesta, Ammar, \& Cueto, 2010; Chinesta, Ladevèze, Ammar, Cueto, \& Nouy, 2009; Ladevèze, Passieux, \& Néron, 2009; Nouy, 2010). This method is based on a representation of the solution using separated variables functions (or modes); this kind of representation, initially derived from Proper Orthogonal Decomposition (POD), enables a linear increase of the number of unknowns with respect to the number of parameters in the problem. However, contrary to POD, the originality of PGD is that it does not lean upon a projection basis that would be a priori given (the construction of such a basis requiring a knowledge, at least partial, of the solution of the problem); in the PGD, an iterative strategy is set up and modes are computed on the fly along iterations by solving simple one variable problems.

*Corresponding author. Email: chamoin@1mt.ens-cachan.fr 
Despite numerous advantages of PGD, a major limitation resides in the fact that there is currently no robust verification tool in order to measure the quality of the obtained approximate PGD solution. Basic results on a priori error estimation for representations by means of separated variables functions can be found in Ladevèze (1998), whereas a first attempt for a posteriori error estimation in the PGD framework was proposed in Ammar, Chinesta, Diez, \& Huerta (2010). Nevertheless, those works do not provide guaranteed and accurate error estimates, which is a serious drawback for relevant design using PGD.

In this work, we introduce a robust error estimation method for numerical simulations based on PGD (Ladevèze \& Chamoin, 2011). The estimator is based on the concept of constitutive relation error (CRE) and associated admissible fields (Ladevèze \& Leguillon, 1983; Ladevèze \& Rougeot, 1997; Ladevèze \& Pelle, 2004). Considering transient thermics problems, we show that the construction of admissible fields can be performed by means of a double PGD approach: (i) a classical kinematic approach in order to construct a temperature field which is kinematically admissible (KA) and (ii) a (non-classical) dual approach in order to build a flux field which is statically admissible (SA). Nevertheless, both approaches lead to problems that can be easily solved with standard tools available in commercial codes; this makes the proposed error estimation method practical and relevant for industrial applications.

The estimate takes all error sources into account - that are, in our case, space and time discretisations - as well as the truncation of the series in the modal representation. Furthermore, specific indicators are introduced in order to split error contributions among all sources and drive adaptive algorithms effectively. Eventually, adjoint-based techniques are introduced in order to use the estimate based on CRE for goal-oriented error estimation, i.e. control of the error on outputs of interest which may be relevant for design purposes.

The paper is organised as follows: after this introduction, the reference transient thermics problem as well as its PGD approximation are presented in Section 2; Section 3 is devoted to the construction of admissible fields in the PGD framework; global and goal-oriented error estimators based on CRE, as well as the construction of error indicators, are then introduced in Section 4 and numerical results on 2D applications are shown in Section 5, before drawing concluding remarks in Section 6.

\section{Reference problem and PGD approximation}

\subsection{Reference problem}

We consider a transient thermics problem on a given structure represented by an open bounded domain $\Omega$, with boundary $\partial \Omega$, and over the time interval $\mathcal{I}=[0, T]$. Structure $\Omega$ is subjected to a given temperature (supposed zero here) on boundary $\partial_{u} \Omega \subset \partial \Omega\left(\partial_{u} \Omega \neq \emptyset\right)$, and to a time-dependent given thermal loading: (i) a thermal flux $q_{\mathrm{d}}(\mathbf{x}, t)$ on $\partial_{q} \Omega \subset \partial \Omega$, with $\partial_{u} \Omega \cap \partial_{q} \Omega=\emptyset$ and $\overline{\partial_{u} \Omega \cup \partial_{q} \Omega}=\partial \Omega$ and (ii) a source term $f_{\mathrm{d}}(\mathbf{x}, t)$ in $\Omega$. The material is assumed to be isotropic, with diffusion coefficients $\mu$, density $\rho$ and thermal capacity $c$. Initial conditions are considered equal to zero. The associated problem consists of finding the temperature-flux pair $(u(\mathbf{x}, t), \mathbf{q}(\mathbf{x}, t))$, with $(\mathbf{x}, t) \in \Omega \times \mathcal{I}$, verifying:

- thermal constraints:

$$
u=0 \quad \text { on } \partial_{u} \Omega \times \mathcal{I}
$$


- equilibrium equations:

$$
\rho c \frac{\partial u}{\partial t}=-\nabla \cdot \mathbf{q}+f_{\mathrm{d}} \quad \text { in } \Omega \times \mathcal{I} ; \quad \mathbf{q} \cdot \mathbf{n}=q_{\mathrm{d}} \quad \text { on } \partial_{q} \Omega \times \mathcal{I}
$$

- constitutive relation:

$$
\mathbf{q}=-\mu \nabla u \quad \text { in } \Omega \times \mathcal{I}
$$

- initial conditions:

$$
u\left(\mathbf{x}, 0^{+}\right)=0 \quad \forall \mathbf{x} \in \Omega
$$

$n$ denotes the outgoing normal to $\Omega$. In the following, the change of variable $\mathbf{q} \rightarrow-\mathbf{q}$ is done so that the constitutive relation reads $\mathbf{q}=\mu \nabla u$.

Introducing functional spaces $\mathcal{V}$ and $\mathcal{T}$ that ensure the regularity required in space and time, respectively, the weak formulation of the problem reads: find solution $u \in \mathcal{V} \otimes \mathcal{T}$ such that:

$$
B(u, v)=L(v) \quad \forall v \in \mathcal{V} \otimes \mathcal{T}
$$

where $B(\cdot, \cdot)$ and $L(\cdot)$ are bilinear and linear forms defined as:

$$
\begin{aligned}
B(u, v) & =\int_{0}^{T} b(u, v) \mathrm{d} t+\int_{\Omega} u\left(\mathbf{x}, 0^{+}\right) v\left(\mathbf{x}, 0^{+}\right) \mathrm{d} \Omega \\
L(v) & =\int_{0}^{T} l(v) \mathrm{d} t \text { with } b(u, v)=\int_{\Omega}\left\{\rho c \frac{\partial u}{\partial t} v+\mu \nabla u \cdot \nabla v\right\} \mathrm{d} \Omega \text { and } \\
l(v) & =\int_{\Omega} f_{\mathrm{d}} v \mathrm{~d} \Omega-\int_{\partial_{q} \Omega} q_{\mathrm{d}} v \mathrm{~d} S .
\end{aligned}
$$

The exact solution of (5), which is generally unreachable, is denoted $\left(u_{\mathrm{ex}}, \mathbf{q}_{\mathrm{ex}}\right)$. Eventually, we introduce the following inner products and norms, for functions $u$ and $v$ in $\mathcal{V} \otimes \mathcal{T}$ :

$$
\begin{gathered}
\langle u, v\rangle_{\Omega}=\int_{\Omega} u v \mathrm{~d} \Omega ; \quad\langle u, v\rangle_{\mathcal{I}}=\int_{0}^{T} u v \mathrm{~d} t ; \quad\langle\langle u, v\rangle\rangle=\int_{0}^{T} \int_{\Omega} u v \mathrm{~d} \Omega \mathrm{d} t \\
\|v\|_{\Omega}=\sqrt{\langle v, v\rangle_{\Omega}} ; \quad\|v\|_{\mathcal{I}}=\sqrt{\langle v, v\rangle_{\mathcal{I}}} ; \quad\|v\| \|=\sqrt{\langle\langle v, v\rangle\rangle}
\end{gathered}
$$

\subsection{Approximation using $P G D$}

In model reduction methods based on separated variables representation (variables being $x$ and $t$ here), the idea is to construct an approximation of $u(x, t)$ as a series of $m$ modes, i.e. products of space and time functions:

$$
u(\mathbf{x}, t) \approx u_{m}(\mathbf{x}, t)=\sum_{i}^{m} \psi_{i}(\mathbf{x}) \lambda_{i}(t)=\Psi_{m}(\mathbf{x}) \cdot \Lambda_{m}(t)
$$


where $\Psi_{m}(\mathbf{x})=\left[\psi_{1}(\mathbf{x}), \psi_{2}(\mathbf{x}), \ldots, \psi_{m}(\mathbf{x})\right]^{T} \quad\left(\right.$ resp. $\left.\quad \Lambda_{m}(t)=\left[\lambda_{1}(t), \lambda_{2}(t), \ldots, \lambda_{m}(t)\right]^{T}\right) \quad$ is $\quad$ a reduced basis of space (resp. time) functions and $m \in \mathbb{N}$ is the representation order. In the PGD approach (Chinesta et al., 2009, 2010; Ladevèze et al., 2009; Nouy, 2010), neither functions $\lambda_{i}(t)$ nor functions $\psi_{i}(\mathbf{x})$ are given initially, and both families are computed on the fly.

We describe here a classical procedure for PGD, called progressive Galerkin. We assume that a separated variables representation $u_{m-1}=\Psi_{m-1} \cdot \Lambda_{m-1}$ of order $m-1$ is known. A new couple $(\psi, \lambda) \in \mathcal{V} \times \mathcal{T}$ is defined for the order $m$ representation as the one that verifies the following double Galerkin orthogonality criterion:

$$
B\left(u_{m-1}+\psi \lambda, \psi \lambda^{*}+\psi^{*} \lambda\right)=L\left(\psi \lambda^{*}+\psi^{*} \lambda\right) \quad \forall \lambda^{*} \in \mathcal{T}, \forall \psi^{*} \in \mathcal{V}
$$

We can thus define the two following applications:

- $S_{m}: \mathcal{T} \rightarrow \mathcal{V}$ is the application that maps a time function $\lambda$ into a space function $\psi=S_{m}(\lambda)$ defined as:

$$
B\left(u_{m-1}+\psi \lambda, \psi^{*} \lambda\right)=L\left(\psi^{*} \lambda\right) \quad \forall \psi^{*} \in \mathcal{V}
$$

It is associated to a (time-dependent) space problem.

- $T_{m}: \mathcal{V} \rightarrow \mathcal{T}$ is the application that maps a space function $\psi$ into a time function $\lambda=T_{m}(\psi)$ defined as:

$$
B\left(u_{m-1}+\psi \lambda, \psi \lambda^{*}\right)=L\left(\psi \lambda^{*}\right) \quad \forall \lambda^{*} \in \mathcal{T}
$$

It is associated to a time problem (scalar ODE).

A couple $(\psi, \lambda)$ verfiies (9) if and only if $\psi=S_{m}(\lambda)$ and $\lambda=T_{m}(\psi)$, which is a nonlinear problem. This version of PGD can then be interpreted as a pseudo eigenvalue problem, functions $\psi$ and $\lambda$, respectively, being dominating eigenfunctions of applications $G_{m}:=S_{m} \circ T_{m}$ and $\tilde{G}_{m}:=T_{m} \circ S_{m}$ (Nouy, 2010). This interpretation is useful as it enables to propose dedicated algorithms, inspired from those used in eigenvalue problems, to build the PGD representation. One of these algorithms, denoted power iterations algorithm, is described below:

$\rightarrow$ for $m=1$ to $m_{\max }$

$\rightarrow$ define $\lambda^{(0)}$ (initialisation)

$\rightarrow$ for $k=1$ to $k_{\text {max }}$

$\rightarrow$ compute $\psi^{(k)}=S_{m}\left(\lambda^{(k-1)}\right)$

$\rightarrow$ normalise $\psi^{(k)}\left(\left\|\psi^{(k)}\right\|_{\Omega}=1\right)$

$\rightarrow$ compute $\lambda^{(k)}=T_{m}\left(\psi^{(k)}\right)$

$\rightarrow$ cheque convergence of $\left(\psi^{(k)} \lambda^{(k)}\right)$

$\rightarrow$ end for

$\rightarrow$ define $\psi_{m}=\psi^{(k)}$ and $\lambda_{m}=\lambda^{(k)}$

$\rightarrow$ define $u_{m}=u_{m-1}+\psi_{m} \lambda_{m}$ and check convergence

$\rightarrow$ end for

We therefore remark that $u_{m}$ is obtained from the solution of a few space or time problems, without any knowledge on $u$; the first step $\psi^{(k)}=S_{m}\left(\lambda^{(k-1)}\right)$ of the iterative strategy, which is the most costly, consists of solving a space problem with fixed time function $\lambda^{(k-1)}$; the sec- 
ond step $\lambda^{(k)}=T_{m}\left(\psi^{(k)}\right)$ consists of solving a time problem over the whole domain $\mathcal{I}$, with fixed space function $\psi^{(k)}$. In practice, both problems are solved with classical numerical methods, i.e. the finite element method (FEM) and a given time integration scheme.

At a given order $m$, space function $\psi_{m}$ can be orthogonalised with respect to the existing space basis $\left\{\psi_{1}, \psi_{2}, \ldots, \psi_{m-1}\right\}$; this procedure generally gives better results. Another simple modification in the power iterations algorithm was proposed to construct a better PGD representation with reasonable computational cost (Ladevèze et al., 2009; Nouy, 2010). It consists of introducing application $T$ in order to update the whole set of time functions $\Lambda_{m}$ after each construction of a new couple $\left(\psi_{m}, \lambda_{m}\right)$. This leads to the following algorithm:

$\rightarrow$ for $\mathrm{m}=1$ to $m_{\max }$

$\rightarrow$ do steps 2 to 8 of the classical power iterations algorithm

$\rightarrow$ define $\psi_{m}=\psi^{\left(k_{\max }\right)}$

$\rightarrow$ compute $\Lambda_{m}=T\left(\Psi_{m}\right)$

$\rightarrow$ define $u_{m}=\Psi_{m} \cdot \Lambda_{m}$ and check convergence

$\rightarrow$ end for

\section{Construction of admissible fields in the PGD framework}

Considering the diffusion problem introduced in Section 2.1, we present two PGD approaches (kinematic and static) that are the basis to derive admissible fields.

\subsection{Kinematic approach}

We consider the weak form (5). In order to compute an approximation of $u$, we use the progressive PGD approach defined in Section 2.2. Knowing $u_{m-1}$, this approach first consists of finding the pair $(\psi, \lambda)$ such that:

$$
B\left(\psi \lambda, \psi \lambda^{*}+\psi^{*} \lambda\right)=R_{m-1}\left(\psi \lambda^{*}+\psi^{*} \lambda\right) \quad \forall \lambda^{*} \in \mathcal{T}, \forall \psi^{*} \in \mathcal{V}
$$

where $R_{m-1}(\cdot)=L(\cdot)-B\left(u_{m-1}, \cdot\right)$ is the order $m-1$ residual; this problem is solved using the power iterations algorithm. After convergence of $\left(\psi^{(k)}, \lambda^{(k)}\right)$, we take $\psi_{m}=\psi^{(k)}$ and we update all time functions by means of the application $\Lambda_{m}=T\left(\Psi_{m}\right)$. We then obtain the order $m$ PGD approximation $u_{m}$ of $u$ :

$$
u_{m}=\Psi_{m} \cdot \Lambda_{m}
$$

In practice, the power iterations algorithm requires the solution of two kinds of problems:

- the application $\psi=S_{m}(\lambda)$ leads to a space problem of the type: find $\psi \in \mathcal{V}$ such that

$$
\int_{\Omega}\left\{\alpha_{S} \rho c \psi \psi^{*}+\beta_{S} \mu \nabla \psi \cdot \nabla \psi^{*}\right\} \mathrm{d} \Omega=\bar{l}\left(\psi^{*}\right)-\sum_{i=1}^{m-1} \int_{\Omega}\left\{\alpha_{S, i} \rho c \psi_{i} \psi^{*}+\beta_{S, i} \mu \nabla \psi_{i} \cdot \nabla \psi^{*}\right\} \mathrm{d} \Omega
$$

with

$$
\begin{gathered}
\alpha_{S}=\langle\dot{\lambda}, \lambda\rangle_{\mathcal{I}} ; \quad \beta_{S}=\|\lambda\|_{\mathcal{I}}^{2} ; \quad \bar{l}(\cdot)=\langle l(\cdot), \lambda\rangle_{\mathcal{I}} \\
\alpha_{S, i}=\left\langle\dot{\lambda}_{i}, \lambda\right\rangle_{\mathcal{I}} ; \quad \beta_{S, i}=\left\langle\lambda_{i}, \lambda\right\rangle_{\mathcal{I}}
\end{gathered}
$$


An approximate solution $\psi^{h}$ of $\psi$ is computed using the FEM with finite element space $\mathcal{V}^{h} \subset \mathcal{V}$, leading to a discrete system of the form:

$$
\left(\alpha_{S} \mathbb{M}+\beta_{s} \mathbb{K}\right) \mathrm{X}=\mathrm{F}
$$

- the application $\lambda=T_{m}(\psi)$ leads to a time problem of the type: find $\lambda \in \mathcal{T}$ such that

$$
\lambda(0)=0 ; \quad \alpha_{T} \dot{\lambda}+\beta_{T} \lambda=\delta_{T}
$$

with

$$
\alpha_{T}=\int_{\Omega} \rho c \psi^{2} \mathrm{~d} \Omega ; \quad \beta_{T}=\int_{\Omega} \mu \nabla \psi \cdot \nabla \psi \mathrm{d} \Omega ; \quad \delta_{T}=l(\psi)-b\left(u_{m-1}, \psi\right)
$$

An approximate solution $\lambda^{\Delta t}$ of $\lambda$ is computed using a given time integration scheme.

We finally obtain an order $m$ PGD approximation $u_{m}^{h, \Delta t}=\sum_{i=1}^{m} \psi_{i}^{h}(\mathbf{x}) \lambda_{i}^{\Delta t}(t)$. It is $K A$ in the sense that it verifies Equations (1) and (4) of the reference problem. However, the associated couple $\left(u_{m}^{h, \Delta t}, \mathbf{q}\left(u_{m}^{h, \Delta t}\right)\right)$, with $\mathbf{q}\left(u_{m}^{h, \Delta t}\right)=\mu \nabla u_{m}^{h, \Delta t}$, is usually not SA as it does not verify the equilibrium (2), even in the following finite element weak sense:

$$
\int_{\Omega} \mathbf{q}\left(u_{m}^{h, \Delta t}\right) \cdot \nabla v^{*} \mathrm{~d} \Omega=l\left(v^{*}\right)-\int_{\Omega} \rho c \frac{\partial u_{m}^{h, \Delta t}}{\partial t} v^{*} \mathrm{~d} \Omega \quad \forall v^{*} \in \mathcal{V}^{h}, \forall t \in \mathcal{I}
$$

In the next section, we introduce a static PGD approach that provides for a flux that verifies this last equilibrium.

\subsection{Static approach}

We define an order $m$ PGD approximation of the flux as:

$$
\mathbf{q}_{m}(\mathbf{x}, t)=\mathbf{q}_{0}^{h}(\mathbf{x}, t)+\sum_{i=1}^{m} \phi_{i}(\mathbf{x}) \xi_{i}(t)=\mathbf{q}_{0}^{h}(\mathbf{x}, t)+\Phi_{m}(\mathbf{x}) \times \Xi_{m}(t)
$$

where $\Phi_{m}(\mathbf{x})=\left[\phi_{1}(\mathbf{x}), \phi_{2}(\mathbf{x}), \ldots, \phi_{m}(\mathbf{x})\right]^{T}$ and $\Xi_{m}(t)=\left[\xi_{1}(t), \xi_{2}(t), \ldots, \xi_{m}(t)\right]^{T}$ are reduced bases of space and time functions, respectively. $\mathbf{q}_{0}^{h}(\mathbf{x}, t)$ is a particular flux in equilibrium with the thermal loading in a finite element sense, and space functions $\phi_{i}(\mathbf{x})$ are SA to zero (SA0) in a finite element sense, i.e. they verify:

$$
\int_{\Omega} \phi_{i} \cdot \nabla v^{*} \mathrm{~d} \Omega=0 \quad \forall v^{*} \in \mathcal{V}^{h}
$$

The associated space is denoted as $\mathcal{Q}_{0}^{h}$.

On the one hand, assuming that the external loading $\left(f_{\mathrm{d}}, q_{\mathrm{d}}\right)$ can be written using the radial approximation,

$$
f_{\mathrm{d}}(\mathbf{x}, t)=F_{\mathrm{d}}(\mathbf{x}) \kappa_{\mathrm{f}}(t) ; \quad q_{\mathrm{d}}(\mathbf{x}, t)=Q_{\mathrm{d}}(\mathbf{x}) \kappa_{q}(t)
$$

the computation of $\mathbf{q}_{0}^{h}(\mathbf{x}, t)$ can be easily performed using a classical finite element procedure. 
On the other hand, noticing that the dual Galerkin formulation of the diffusion problem consists of finding $\mathbf{q} \in \mathcal{Q} \otimes \mathcal{T}$ such that:

$$
\int_{0}^{T} \int_{\Omega} \frac{1}{\mu} \mathbf{q} \cdot \mathbf{q}^{*} \mathrm{~d} \Omega \mathrm{d} t=0 \quad \forall \mathbf{q}^{*} \in \mathcal{Q}_{0}
$$

and assuming that $\mathbf{q}_{m-1}=\mathbf{q}_{0}^{h}+\sum_{i=1}^{m-1} \phi_{i} \xi_{i}$ is known, the progressive PGD at order $m$ (with updating of time functions) first consists of searching a couple $(\phi, \xi)$ that verifies:

$$
\int_{0}^{T} \int_{\Omega}\left(\mathbf{q}_{m-1}+\phi \xi\right) \cdot \frac{1}{\mu}\left(\phi^{*} \xi+\phi \xi^{*}\right) \mathrm{d} \Omega \mathrm{d} t=0 \quad \forall \phi^{*} \in \mathcal{Q}_{0}^{h}, \forall \xi^{*} \in \mathcal{T}
$$

As for the kinematic approach, the power iterations algorithm requires solving two kinds of problems:

- a space problem of the form $\phi=\tilde{S}_{m}(\xi)$ such that:

$$
\int_{\Omega} \frac{1}{\mu}\{\alpha \phi+\beta\} \cdot \phi^{*} \mathrm{~d} \Omega=0 \quad \forall \phi^{*} \in \mathcal{Q}_{0}^{h}
$$

with $\alpha=\|\xi\|_{\mathcal{I}}^{2}$ and $\beta=\left\langle\xi, \mathbf{q}_{m-1}\right\rangle_{\mathcal{I}}$. Using duality arguments, one shows that there is a function $w^{h}(\mathbf{x}) \in \mathcal{V}^{h}$ such that:

$$
\alpha \phi+\beta=\mu \nabla w^{h}
$$

Therefore, $w^{h}$ should verify:

$$
\int_{\Omega} \frac{1}{\alpha}\left\{\mu \nabla w^{h}-\beta\right\} \cdot \nabla w^{*} \mathrm{~d} \Omega=0 \quad \forall w^{*} \in \mathcal{V}^{h}
$$

and this last problem can be solved with the standard FEM.

- a time problem of the form $\xi=\tilde{T}_{m}(\phi)$ such that:

$$
\int_{\Omega} \frac{1}{\mu}\left(\mathbf{q}_{m-1}+\phi \xi\right) \cdot \phi \mathrm{d} \Omega=0 \quad \forall t \in \mathcal{I}
$$

This provides for the explicit expression of $\xi(t)$ (after normalisation of $\phi$ );

$$
\xi(t)=-\left\langle\mathbf{q}_{m-1}(\cdot, t), \phi\right\rangle_{\Omega}
$$

In a second stage, vector $\Xi_{m}(t)$ of time functions is updated using global application $\Xi_{m}=\tilde{T}\left(\Phi_{m}\right)$. The power iterations algorithm that is employed to compute the static PGD version of $\mathbf{q}$ is the same as the one previously described for $u$.

Of course, space or time problems associated to applications $\tilde{S}_{m}$ and $\tilde{T}_{m}$ are again solved approximately, and the computed PGD representation is denoted $\mathbf{q}_{m}^{h, \Delta t}=\mathbf{q}_{0}^{h}+\sum_{i=1}^{m} \phi_{i}^{h} \xi_{i}^{\Delta t}$.

Consequently, couple $\left(u_{m}^{h, \Delta t}, \mathbf{q}_{m}^{h, \Delta t}\right)$ verifies (19) i.e. it is SA in the finite element sense. 


\subsection{Computation of admissible fields}

The error estimator that we set up is based on the CRE (Ladevèze \& Pelle, 2004). The use of CRE requires the calculation of an admissible temperature-flux couple, denoted $\left(\hat{u}^{h, \Delta t}, \hat{\mathbf{q}}^{h, \Delta t}\right)$ in the following, associated to the computed PGD solution. Couple $\left(\hat{u}^{h, \Delta t}, \hat{\mathbf{q}}^{h, \Delta t}\right)$ is admissible in the sense that it verifies (1), (2) and (4). Its construction is performed from the approximate solution $\left(u_{m}^{h, \Delta t}, \mathbf{q}_{m}^{h, \Delta t}\right)$ at hand:

- a temperature field $\hat{u}^{h, \Delta t}$ verifying (1) and (4) is said KA; a simple choice consists of taking the temperature field $u_{m}^{h, \Delta t}$ coming the kinematic PGD approach;

- a couple $\left(\hat{u}^{h, \Delta t}, \hat{\mathbf{q}}^{h, \Delta t}\right)$ verifying (2) is said SA; the construction of $\hat{\mathbf{q}}^{h, \Delta t}$ is the major technical point. It consists of using an energy relation, named prolongation condition, implying field $\mathbf{q}_{m}^{h, \Delta t}$ coming from the static PGD approach and using its properties; this leads to the solution of local problems defined over each element of the mesh. Full details on some techniques usable to construct $\hat{\mathbf{q}}^{h, \Delta t}$ at each time point can be found in Ladevèze and Pelle (2004).

Remark. In practice, $\hat{\mathbf{q}}_{m}^{h, \Delta t}$ is constructed as:

$$
\hat{\mathbf{q}}_{m}^{h, \Delta t}=\hat{\mathbf{q}}_{0}^{h}+\sum_{i=1}^{m} \hat{\boldsymbol{\phi}}_{i}^{h} \xi_{i}^{\Delta t} ; \quad \hat{\mathbf{q}}_{0}^{h}=\hat{\mathbf{q}}\left(z_{\mathrm{f}}^{h}\right) \kappa_{f}(t)+\hat{\mathbf{q}}\left(z_{q}^{h}\right) \kappa_{q}(t)+\sum_{i=1}^{m} \hat{\mathbf{q}}\left(s_{i}^{h}\right) \dot{\lambda}_{i}^{\Delta t}(t)
$$

where $\hat{\boldsymbol{\phi}}_{0}^{h}$ and $\hat{\mathbf{f}}_{i}^{h}$ are SA and SA0 fields, verifying the prolongation condition with $\mathbf{q}_{0}^{h}$ and $\phi_{i}^{h}$, respectively.

The computational cost associated to the construction of $\hat{\mathbf{q}}_{m}^{h, \Delta t}$ is $(m+2) \times\left(C_{\mathrm{v}} N_{\mathrm{v}}+\right.$ $C_{\mathrm{e}} N_{\mathrm{e}}$ ), where $N_{\mathrm{v}}$ (resp. $N_{\mathrm{e}}$ ) is the number of vertices (resp. elements) in the mesh and $C_{\mathrm{v}}$ (resp. $C_{\mathrm{e}}$ ) is the computational cost associated to a problem on a patch of elements (resp. to a problem at the element level).

\section{Global and goal-oriented error estimation}

\subsection{Global error estimation}

From any admissible solution $(\hat{u}, \hat{\mathbf{q}})$ (previous calculations lead to take $(\hat{u}, \hat{\mathbf{q}})=\left(\hat{u}_{m}^{h, \Delta t}, \hat{\mathbf{q}}_{m}^{h, \Delta t}\right)$ ), the CRE is defined as a measure $E_{\mathrm{CRE}}$ of the non-verification of (3) by the admissible couple. It reads:

$$
E_{\mathrm{CRE}}^{2}(\hat{u}, \hat{\mathbf{q}}) \equiv \int_{0}^{T} \int_{\Omega}(\hat{\mathbf{q}}-\mu \nabla \hat{u}) \cdot \frac{1}{\mu}(\hat{\mathbf{q}}-\mu \nabla \hat{u}) \mathrm{d} \Omega \mathrm{d} t=\|\hat{\mathbf{q}}-\mu \nabla \hat{u}\| \|_{\mu^{-1}}^{2}
$$

A fundamental property of CRE is its link with the exact solution $\left(u^{\mathrm{ex}}, \mathbf{q}^{\mathrm{ex}}\right)$ :

$$
\left\|\mid u^{\mathrm{ex}}-\hat{u}\right\|\left\|_{\mu}^{2}+\right\|\left\|\mathbf{q}^{\mathrm{ex}}-\hat{\mathbf{q}}\right\|_{\mu^{-1}}^{2}+\mathcal{G}\left(u^{\mathrm{ex}}-\hat{u}\right)=E_{\mathrm{CRE}}^{2}(\hat{u}, \hat{\mathbf{q}})
$$

with $\|\cdot\| \|_{\mu}^{2}=\int_{0}^{T} \int_{\Omega} \mu \nabla(\cdot) \cdot \nabla(\cdot) \mathrm{d} \Omega \mathrm{d} t$ and $\mathcal{G}\left(u^{\mathrm{ex}}-\hat{u}\right)=\int_{\Omega} \rho c\left(u^{\mathrm{ex}}-\hat{u}\right)_{\mid t=T}^{2} \mathrm{~d} \Omega \geq 0$.

Equation (32), that can be seen as an extension of Prager-Synge equality for time-dependent problems, directly shows that $\left\|\mid u^{\mathrm{ex}}-\hat{u}\right\|_{\mu} \leq E_{\mathrm{CRE}}$ so that measure $E_{\mathrm{CRE}}$ is a guaranteed upper bound of the error (given in the energy norm). It takes all error sources into account, i.e. time and space discretisations as well as truncation of the series in the PGD representation 
when choosing $(\hat{u}, \hat{\mathbf{q}})=\left(\hat{u}_{m}^{h, \Delta t}, \hat{\mathbf{q}}_{m}^{h, \Delta t}\right)$. The computational cost required to compute $E_{\mathrm{CRE}}$ is proportional to $N_{\mathrm{g}} \times\left(N_{\mathrm{p}}+1\right)$ where $N_{\mathrm{g}}$ (resp. $\left.N_{\mathrm{p}}\right)$ is the number of Gauss points in the mesh (resp. number of time steps).

\subsection{Error indicators}

In order to optimise the adaptive process that would aim at decreasing error ||$\left|u^{\mathrm{ex}}-\hat{u}_{m}^{h, \Delta t}\right| \mid \|_{\mu}$, one must be able to assess contributions of various error sources; this can be done defining appropriate error indicators. The procedure to define such indicators is very simple: we first define solution $u^{h, \Delta t}$ of the discrete problem obtained after discretising the initial thermics problem in space and time (with the same discretisations as those used to compute $u_{m}^{h, \Delta t}$ ). Using for instance the FEM and a forward Euler time scheme, $u^{h, \Delta t}$ is obtained solving a system of the form ( $P$ being the number of time steps):

$$
\mathbf{U}_{h}^{1}=\mathbf{0} ; \quad \mathbb{M} \frac{\mathbf{U}_{h}^{p+1}-\mathbf{U}_{h}^{p}}{\Delta t}+\mathbb{K} \mathbf{U}_{h}^{p}=\phi_{h}^{p} \quad \forall p \in[1, P-1]
$$

We can then write:

$$
\left\|\left.\left|u^{\mathrm{ex}}-u_{m}^{h, \Delta t}\right|\right|_{\mu} ^{2}=\left|\left\|u^{\mathrm{ex}}-u^{h, \Delta t}||_{\mu}^{2}+\right\|\right| u^{h, \Delta t}-u_{m}^{h, \Delta t}||_{\mu}^{2}\right.
$$

where $\left\|\left|u^{\mathrm{ex}}-u^{h, \Delta t}\right|\right\|_{\mu}$ is the contribution to the error coming from time and space discretisations alone and ||$\left|u^{h, \Delta t}-u_{m}^{h, \Delta t}\right| \|_{\mu}$ is the contribution to the error coming from the PGD representation alone. The second contribution can be directly assessed in the post-process, without any additional computation, defining problem (33) as a new reference problem and using the CRE. In this framework, an associated admissible couple $\left(\hat{u}^{h, \Delta t}, \hat{\mathbf{q}}^{h, \Delta t}\right)$ (in the sense of (33)) can be defined; it is directly obtained at each time point as a post-processing of solution $\left(u_{m}^{h, \Delta t}, \mathbf{q}_{m}^{h, \Delta t}\right)$ at hand. One can thus compute the estimate:

$$
E_{\mathrm{CRE}, \mathrm{PGD}}=\left\|\left|\hat{\mathbf{q}}^{h, \Delta t}-\mu \nabla \hat{u}^{h, \Delta t}\right|\right\|_{\mu^{-1}}
$$

of error ||$\left|u^{h, \Delta t}-u_{m}^{h, \Delta t}\right| \|_{\mu}$ coming from the PGD representation.

In a second stage, (34) enables to define the estimate $E_{\mathrm{CRE}, \text { dis }}$ of error $\left\|\left|u^{\mathrm{ex}}-u^{h, \Delta t}\right|\right\|_{\mu}$ coming from time and space discretisations; it reads:

$$
E_{\mathrm{CRE}, \mathrm{dis}}=\sqrt{E_{\mathrm{CRE}}^{2}-E_{\mathrm{CRE}, \mathrm{PGD}}^{2}}
$$

Comparison between values of $E_{\mathrm{CRE}, \text { dis }}$ and $E_{\mathrm{CRE}, \mathrm{PGD}}$ enables to choose the critical error source and lead the adaptive process effectively.

\subsection{Goal-oriented error estimation}

We now deal with estimation of the error on specific outputs of interest. Such local quantities, denoted $I$ in the following, can be written under a global form:

$$
I=\int_{0}^{T} \int_{\Omega}\left(\mathbf{q}_{\Sigma} \cdot \nabla u+f_{\Sigma} u\right) \mathrm{d} \Omega \mathrm{d} t
$$


where $\mathbf{q}_{\Sigma}$ and $f_{\Sigma}$ are extraction functions (possibly defined as Dirac distributions). A general procedure consists then of introducing the associated adjoint problem that reads: find the temperature-flux pair $(\tilde{u}, \tilde{\mathbf{q}})$ that verifies:

- thermal constraints:

$$
\tilde{u}=0 \quad \text { on } \partial_{u} \Omega \times \mathcal{I}
$$

- equilibrium equations:

$$
\rho c \frac{\partial \tilde{u}}{\partial t}-\nabla \cdot\left(\tilde{\mathbf{q}}-\mathbf{q}_{\Sigma}\right)+f_{\Sigma}=0 \quad \text { in } \Omega \times \mathcal{I} ; \quad \tilde{\mathbf{q}} \cdot \mathbf{n}=0 \quad \text { on } \partial_{q} \Omega \times \mathcal{I}
$$

- constitutive relation:

$$
\tilde{\mathbf{q}}=-\mu \nabla \tilde{u} \quad \text { in } \Omega \times \mathcal{I}
$$

- final conditions:

$$
\tilde{u}(\mathbf{x}, T)=0 \quad \forall \mathbf{x} \in \Omega
$$

This problem, which is similar to the reference problem when performing the change of variable $t^{\prime}=T-t$, is solved using the PGD approach (with $M$ modes) providing for the approximate solution $\left(\tilde{u}_{M}^{h, \Delta t}, \tilde{\mathbf{q}}_{M}^{h, \Delta t}\right)$. Furthermore, admissible fields $\left(\hat{\tilde{u}}_{M}^{h, \Delta t}, \hat{\mathbf{q}}_{M}^{h, \Delta t}\right)$ can be computed using the procedure of Section 3 , which provides for the global error estimate $\tilde{E}_{\mathrm{CRE}}$ for the adjoint problem.

Error estimation on $I$ is then obtained from the following fundamental result, valid for linear elliptic and parabolic problems (see (Ladevèze, 2008)):

$$
\left|I_{\mathrm{ex}}-I_{m}^{h, \Delta t}-I_{\mathrm{corr}}\right| \leq E_{\mathrm{CRE}} \tilde{E}_{\mathrm{CRE}}
$$

where $I_{\mathrm{ex}}$ (resp. $I_{m}^{h, \Delta t}$ ) is the exact (resp. approximated by PGD) value of the output of interest and $I_{\text {corr }}$ is a correction term computed from approximate solutions of both reference and adjoint problems. In order to optimise the quality of error bounds obtained from (42), the PGD adjoint solution is derived introducing locally and enrichment term computed offline (see (Ladevèze \& Chamoin, 2010) for full details).

\section{Numerical results}

In the following numerical experiment, the power iterations algorithm is initialised with $\lambda^{(0)}(t)=t\left(\xi^{(0)}(t)=t\right)$. We use the FEM with linear elements to solve space problems, and a forward Euler scheme to solve time problems. The number of iterations performed in the power iterations algorithm is fixed to $k_{\max }=4$.

We consider the 2D structure of Figure 1, which contains two rectangular holes in which a fluid circulates; using symmetries, we study one quarter (denoted by $\Omega$ ) of the full $2 \mathrm{D}$ domain. It is subjected over $[0, T]$ to a given flux $q_{\mathrm{d}}=-1$ applied on the hole boundary; a 

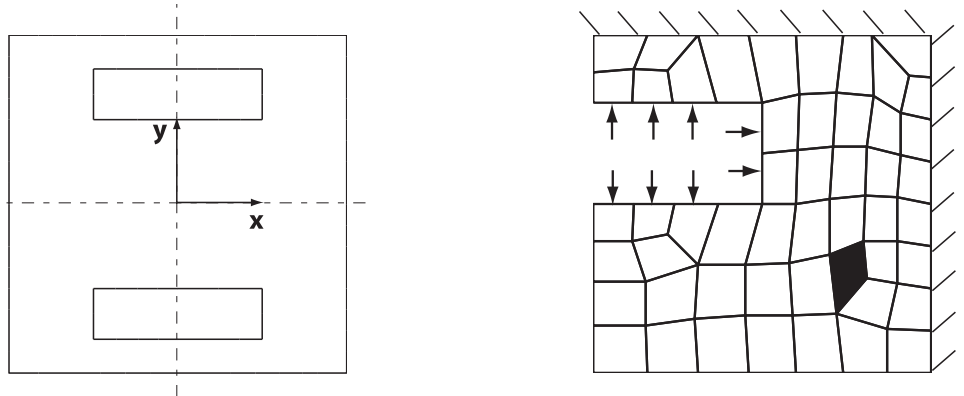

Figure 1. 2D domain considered (left), and associated finite element mesh (right).
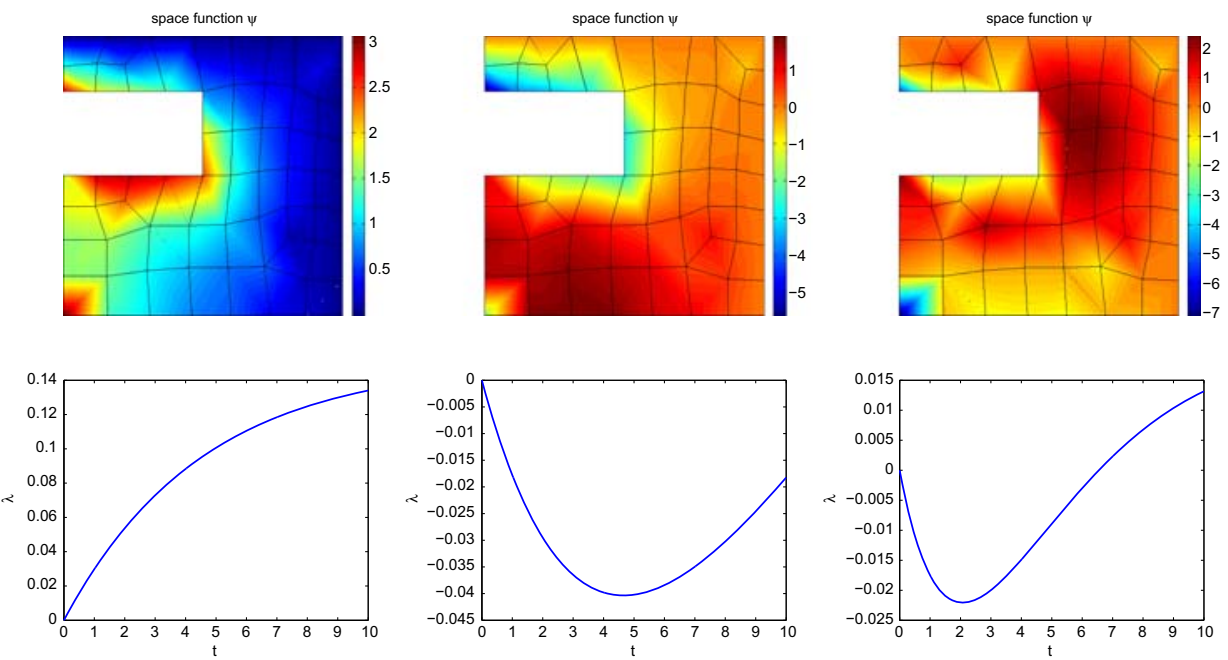

Figure 2. Space functions $\psi_{m}$ (top) and time functions $\lambda_{m}$ (bottom) for $m=1,2,3$ (from left to right).

source term of the form $f_{\mathrm{d}}(x, y)=200 x y$ is also applied in $\Omega$. For the numerical study, we choose $T=10, \mu=1$ and $\rho c=1$.

The space mesh used to discretise $\Omega$ consists of $N_{\mathrm{e}}=50$ quadrangular elements, and time domain $[0, T]$ is divided into $N_{\mathrm{p}}=1000$ time steps.

We represent in Figure 2 first modes $\left(\psi_{m}, \lambda_{m}\right)$ obtained for $m_{\max }=5$.

In Figure 3, the value of the relative error estimate $\bar{E}_{\mathrm{CRE}}=E_{\mathrm{CRE}} /\|\hat{\mathbf{q}} \mid\|_{\mu^{-1}}$ is given with respect to the PGD order $m$ which is used $(m \in[1,10])$. We observe that the error estimate reaches convergence for $m=4$. Let us note that for this value of $m$, the CPU time required to obtain the error estimate (normalised by the time necessary to solve the reference problem using PGD) is 2.8 .

We now introduce the new reference problem obtained after time and space discretisations of the initial reference problem. Computing associated admissible fields, we obtain indicator $E_{\mathrm{CRE}, \mathrm{PGD}}$ and then deduce indicator $E_{\mathrm{CRE} \text {,dis. }}$ The values of three estimates $E_{\mathrm{CRE}}, E_{\mathrm{CRE}, \mathrm{PGD}}$ and $E_{\mathrm{CRE}, \text { dis }}$ are plotted in Figure 3 with respect to order $m$.

We observe that indicator $E_{\mathrm{CRE} \text {,dis }}^{2}$ provides for a relevant assessment of the discretisation error, even for small values of $m$. Furthermore, we can see that indicator $E_{\mathrm{CRE} \text {,dis }}$ becomes 
larger than $E_{\mathrm{CRE}, \mathrm{PGD}}$ for $m \geq 3$; this could be used in an optimised adaptive process in which the number of computed modes would be first increased, up to $m=3$, before refining time and space discretisations in order to improve the quality of the PGD solution.

Now dealing with goal-oriented error estimation, we consider as an output of interest the mean value of $u$ inside a local zone $\omega \subset \Omega$ (black zone in Figure 1) at final time $T$ :

$$
I=\frac{1}{|\omega|} \int_{\omega} u_{\mid T} \mathrm{~d} \Omega
$$

Consequently, the extraction functions read:

$$
\mathbf{q}_{\Sigma}(\mathbf{x}, t)=\mathbf{0} ; \quad f_{\Sigma}(\mathbf{x}, t)=\frac{1}{|\omega|} I_{\omega}(\mathbf{x}) \delta(t-T)
$$

where $I_{\omega}$ is the indicatrix function of $\omega$ and $\delta(t)$ is the Dirac function in time.

The normalised upper bound $E_{\mathrm{CRE}} \tilde{E}_{\mathrm{CRE}} /\left|I_{m}^{h, \Delta t}\right|$ of the error, as well as specific error indicators, are given in Figure 4 with respect to the number $M$ of computed PGD modes for the adjoint solution ( $m$ is fixed to (2) here).
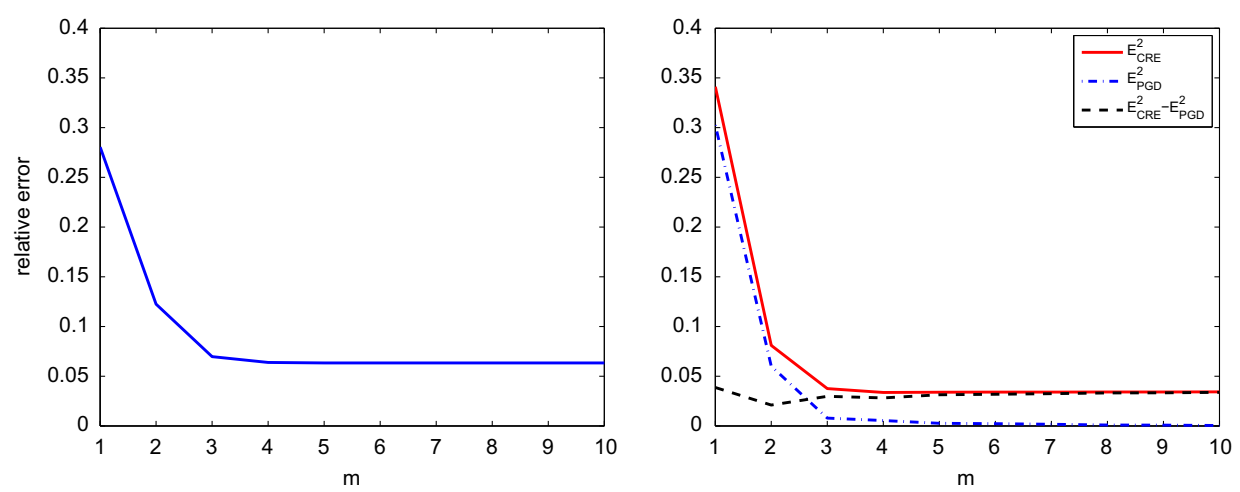

Figure 3. Evolution of estimates $E_{\mathrm{CRE}}^{2}, E_{\mathrm{CRE}, \mathrm{PGD}}^{2}$ and $E_{\mathrm{CRE} \text {,dis }}^{2}$ with respect to PGD order $m$.

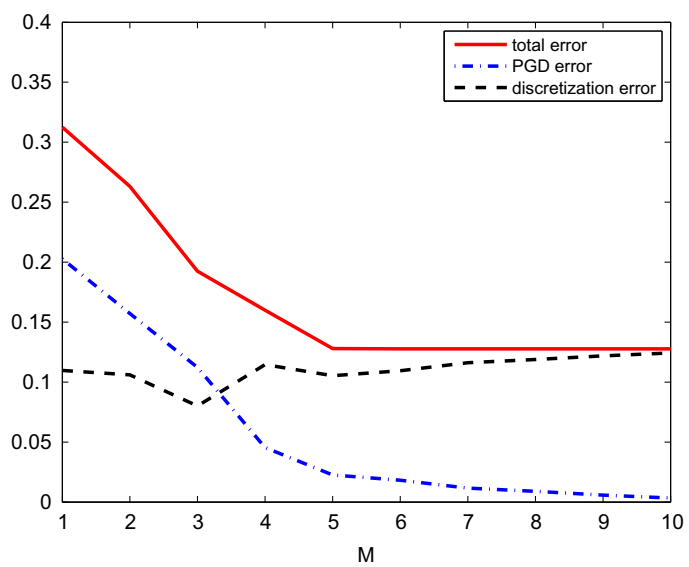

Figure 4. Normalised upper error bound on $I$ and error indicators with respect to $M$. 
We observe that due to the correction term $I_{\text {corr }}$, which has a PGD representation, the quantity $I_{m}^{h, \Delta t}+I_{\text {corr }}$ tends to an asymptotic value when $M$ increases. This value is different from $I_{\mathrm{ex}}$ only due to the discretisation error; therefore, a refinement of space and time discretisations would be necessary to improve the quality of the approximate output of interest in that case.

\section{Conclusions}

We presented a method that provides for a guaranteed and robust error estimator in the framework of numerical simulations performed by means of PGD. This estimator, based on the CRE, uses a double PGD approach of the problem which enables to compute admissible fields with classical tools available in finite element softwares. Indicators on various error sources were also introduced in order to help driving adaptive strategies in an optimal manner, and performances of the CRE estimate to deal with goal-oriented error estimation were analysed.

The error estimation method which is employed here is quite general; it could be used for other multi-parameterised problems such as stochastic problems. This topic is being addressed in ongoing works.

\section{References}

Ammar, A., Chinesta, F., Diez, P., \& Huerta, A. (2010). An error estimator for separated representations of highly multidimensional models. Computer Methods in Applied Mechanics and Engineering, 199, $1872-1880$.

Chinesta F., Ammar A., \& Cueto E. 2010. Recent advances and new challenges in the use of the Proper Generalized Decomposition for solving multidimensional models. Archives of Computational Methods in Engineering, 17(4), 327-350.

Chinesta, F., Ladevèze, P., Ammar, A., Cueto, E., \& Nouy, A. (2009). Proper Generalized Decomposition in extreme simulations: Towards a change of paradigm in computational mechanics? IACM Expressions, 26/09, 2-7.

Ladevèze, P. 1998. Nonlinear computational structural mechanics: New approaches and non-incremental methods of calculation. New York: Springer Verlag.

Ladevèze, P. (2008). Strict upper error bounds for calculated outputs of interest in computational structural mechanics. Computational Mechanics, 42(2), 271-286.

Ladevèze, P., \& Pelle, J.-P. (2004). Mastering calculations in linear and nonlinear mechanics. New York, NY: Springer.

Ladevèze, P., \& Chamoin, L. (2010). Calculation of strict bounds for finite element approximations of non-linear pointwise quantities of interest. International Journal for Numerical Methods in Engineering, 84, 1638-1664.

Ladevèze, P., \& Chamoin, L. (2011). On the control of model reduction methods based on the Proper Generalized Decomposition. Computer Methods in Applied Mechanics and Engineering, 200, 2032 2047.

Ladevèze, P., \& Leguillon, D. (1983). Error estimate procedure in the finite element method and application. SIAM Journal of Numerical Analysis, 20(3), 485-509.

Ladevèze, P., Passieux, J.-C., \& Néron, D. (2009). The LATIN multiscale computational method and the Proper Generalized Decomposition. Computer Methods in Applied Mechanics and Engineering, 199 (21), 1287-1296.

Ladevèze, P., \& Rougeot, P. (1997). New advances on a posteriori error on constitutive relation in finite element analysis. Computer Methods in Applied Mechanics and Engineering, 150, 239-249.

Nouy, A. (2010). A priori model reduction through Proper Generalized Decomposition for solving timedependent partial differential equations. Computer Methods in Applied Mechanics and Engineering, 199(23-24), 1603-1626. 